\title{
Scintigraphic measurement of tracheal mucus velocity in vivo
}

\author{
L. Morgan*, M. Pearson*, R. de longh*, D. Mackey\#, H. van der Wall ${ }^{\#}$, M. Peters*, J. Rutland*
}

Scintigraphic measurement of tracheal mucus velocity in vivo. L. Morgan, M. Pearson, R. de Iongh, D. Mackey, H. van der Wall, M. Peters, J. Rutland. (C) ERS Journals Ltd 2004.

ABSTRACT: Mucociliary clearance (MCC) is an important mechanism for removing inhaled particles, secretions and cellular debris from the respiratory tract. Here, a direct measurement of tracheal mucus velocity (TMV) for assessment of MCC, suitable for clinical and research use, is reported, and a comparison is made of TMV in normal subjects and patients with chronic obstructive pulmonary disease (COPD).

A $0.1-\mathrm{mL}$ bolus of radiolabelled (2-5 MBq), technetium-labelled macroaggregated human albumin $\left({ }^{99 m}\right.$ Tc MAA) was injected through the cricothyroid membrane into the trachea of 20 young $(<50$ yrs) and 12 older $(>50$ yrs) normal subjects and 34 patients with COPD. Repeat studies were carried out in 13 normal subjects and 16 COPD patients. Movement of the bolus in the trachea was recorded $(15 \mathrm{~min})$ using a gamma camera interfaced to a computer. Data were analysed using specifically designed software.

The test was well tolerated by subjects and patients, and no significant adverse events were reported. No significant differences were observed between data recorded from different regions of the bolus (leading edge, peak, trailing edge) by analysis of variance (ANOVA). Bland-Altman plots of the repeat studies indicated that data were more variable in normal subjects (coefficient of repeatability (COR) $10.3 \mathrm{~mm} \cdot \mathrm{min}^{-1}$ ) than in COPD patients (COR $\left.5.5 \mathrm{~mm} \cdot \mathrm{min}^{-1}\right)$. TMV (mean \pm SD) in young normal subjects $(\mathrm{n}=20)$ was $10.7 \pm 3.5 \mathrm{~mm} \cdot \mathrm{min}^{-1}$. TMV was reduced in older normal subjects $(\mathrm{n}=12$; $\left.6.5 \pm 2.6 \mathrm{~mm} \cdot \mathrm{min}^{-1}\right)$ and further reduced in $\operatorname{COPD}\left(\mathrm{n}=34 ; 2.1 \pm 2.7 \mathrm{~mm} \cdot \mathrm{min}^{-1}\right)$.

In conclusion, this technique can be used to measure tracheal mucus velocity rapidly and safely in healthy subjects and patients with respiratory tract disease. This study has confirmed that tracheal mucus velocity declines with age and is further impaired in patients with chronic obstructive pulmonary disease.

Eur Respir J 2004; 23: 518-522.
*Dept of Thoracic Medicine, and " Dept of Nuclear Medicine, Concord Repatriation General Hospital, Concord, and Anatomy and Cell Biology, University of Melbourne, Parkville, Australia.

Correspondence: L. Morgan

Dept of Thoracic Medicine (C31)

Concord Hospital

Hospital Rd

Concord NSW 2139

Australia

Fax: 61297677605

E-mail: morganl@email.cs.nsw.gov.au

Keywords: Chronic obstructive pulmonary disease

cilia

mucociliary clearance

tracheal mucus velocity

Received: July 102002

Accepted after revision: December 82003
Mucociliary clearance (MCC) is an important defence mechanism in the respiratory tract, resulting in the removal of inhaled particles and respiratory secretions. Impaired MCC is known to predispose to bronchial infection and may lead to the development of chronic lung diseases such as bronchiectasis. Measurement of MCC allows a better understanding of such chronic lung diseases and may provide an objective measure of response to therapeutic strategies.

MCC is most commonly measured using inhaled radiolabelled aerosols (IRLA) and scintigraphy. This is a valuable technique but has some limitations. Optimal large airway deposition requires specialised aerosol generation and is affected by airway disease. The acquisition period is relatively long, limiting the use of the test in breathless subjects or those with cough. Data analysis requires correction for isotope decay and signal attenuation. Furthermore, clearance of aerosol occurs in three dimensions and the analysis is based on a two-dimensional representation of the lungs. As a result, the equipment required and the technical features of IRLA studies restrict its use to specialised units.

Some of these problems can be circumvented by direct measurement of particle clearance from the trachea. Measurement of tracheal mucus velocity (TMV) has been investigated using a variety of markers [1-3], deposition techniques [4-6] and detection systems [7, 8]. However, no technique has proved sufficiently practical or reliable for use in clinical practice.

The aim of this project was to develop a simple, reliable and reproducible technique for direct measurement of TMV that could be used easily in a clinical setting. This technique was used to compare TMV in normal subjects with that in subjects with COPD.

\section{Materials and methods}

\section{Subjects}

Twenty young $(<50$ yrs) and 12 older $(>50$ yrs) normal subjects, and 34 patients with COPD were studied. All normal subjects were nonsmokers with no history of lung disease. COPD patients were current or ex-smokers of $\geqslant 20$ pack-yrs, with a forced expiratory volume in one second (FEV1) $<70 \%$ predicted and FEV1/forced vital capacity ratio $<70 \%$ (table 1 ).

Patients were instructed not to use short-acting bronchodilators for $4 \mathrm{~h}$ prior to study. All procedures in this study were performed after obtaining informed consent. The Institutional Ethics Committee of Central Sydney Area Health Service (CRGH Zone) approved the study. 
Table 1.-Mean age and forced expiratory volume in one second $\left(F E V_{1}\right)$ of patient/subject groups

\begin{tabular}{lclc}
\hline & Subjects $n$ & Age yrs & FEV1 \% pred \\
\hline Young normals & 20 & $31.7 \pm 5.0$ & Not measured \\
Older normals & 12 & $66.1 \pm 11.6$ & $91.5 \pm 19.7$ \\
COPD & 34 & $71.5 \pm 7.7$ & $50.9 \pm 13.5$ \\
\hline
\end{tabular}

Data are presented as mean \pm SD. COPD: chronic obstructive pulmonary disease.

\section{Radiolabelling}

Technetium-labelled macroaggregated human albumin $\left({ }^{99 \mathrm{~m}} \mathrm{Tc}\right.$ MAA), in kit form as Technescan $\mathbb{R}$ MAA (Mallinckrodt Medical, Inc., St Louis, MO, USA), was used as the marker. The particle size distribution of the aggregated albumin is such that $\geqslant 90 \%$ are $10-90 \mu \mathrm{m}$ in diameter. Typically, $\sim 90 \%$ are $10-40 \mu \mathrm{m}$ in size.

\section{Bolus droplet deposition}

Each subject was placed in a seated position and the skin overlying the cricothyroid region was anaesthetised with lignocaine cream $(5 \%)$. A $0.1-\mathrm{mL}$ droplet of ${ }^{99 \mathrm{~m}} \mathrm{Tc}$ MAA (2-5 MBq) was injected, using a 25-gauge sterile needle $(0.50 \times 19 \mathrm{~mm})$, attached to a $1-\mathrm{mL}$ tuberculin syringe, held at $\sim 45^{\circ}$ cephalad relative to the trachea, through the cricothyroid membrane. The injection was timed for the end of expiration during tidal breathing. Early experiments, using a clear plastic tracheal model, demonstrated that the combination of the seated position of the subject, gravity and the force of expulsion of the drop from the syringe resulted in deposition onto the posterior surface of the trachea.

\section{Measurement}

After injection, the subject was positioned supine and the gamma camera was positioned above the anterior thorax. This positioning was completed in $<1 \mathrm{~min}$. Images were acquired for $15 \mathrm{~min}$ in the anterior projection on a Siemens Orbiter gamma camera interfaced to an Icon workstation (Siemens, Hoffman Estates, IL, USA) and fitted with a lowenergy all-purpose collimator. The camera setup was standard for ${ }^{99 \mathrm{~m}} \mathrm{Tc}$, with a $15 \%$ window centred at $140 \mathrm{keV}$. A dynamic series of 90 images of $10 \mathrm{~s}$ each were acquired into a $128 \times 128$ matrix.

\section{Analysis}

For each study, the rate of movement of the injected droplet was followed in real time as well as being recorded to permit viewing later as a moving image.

Further analysis of the movement of the peak of activity was made using CiliaCC (RSI Inc., Boulder, CO, USA), a software program written in interactive data language specifically for this project. CiliaC follows the peak of activity by applying a two-dimensional Gaussian fit to each image to find the peak location. Peak positions were plotted as a function of time and then smoothed with a polynomial function. A reference point was selected arbitrarily by the operator on the smoothed curve midway along the steepest part of the slope (the region of highest velocity). CiliaC provides a semiautomated calculation of velocity at that point and then identifies the frame on either side of the reference point at which this slope changes by $>20 \%$. The midpoint between these limits is chosen automatically as a new reference point, and the process is repeated until the start and finish of the region of steepest slope has been delineated.

Maximum velocity was calculated as the gradient of this fitted line. Measurements were accepted only from segments of the trace $>12$ frames $(2 \mathrm{~min})$ in duration.

To determine whether different regions of the bolus moved at different rates over the course of the study, the TMV of the leading and trailing edges were measured, as well as the apparent peak of activity. As analysis of variance showed no significant regional variability, peak activity measurements were used subsequently.

In order to determine the repeatability of this test, repeat TMV measurements of 13 young normal subjects and 16 patients with COPD were carried out at least 1 week after the first study.

\section{Statistics}

All data are expressed as mean \pm SD. Measurements of TMV from different regions of the bolus were compared by onetailed analysis of variance (ANOVA). The repeatability measurements were compared by ANOVA, Bland-Altman agreement plots and by coefficient of repeatability [9]. ANOVA and unpaired t-tests were used to determine the significance of differences in mean TMV between different groups. Differences were considered significant at $\mathrm{p}<0.05$.

\section{Results}

A total of 95 studies were completed (45 in normal subjects and 50 in patients with COPD including repeat studies). All were well tolerated, even in those subjects with severe COPD. One normal subject developed minor bruising at the injection site and two reported minor discomfort on swallowing for up to $12 \mathrm{~h}$ after injection. Cough at the time of injection occurred in five studies and was short lived. There were no other adverse events.

A representative TMV trace showing the movement of the peak of activity of the radiolabelled bolus along the tracheal mucosa of a normal subject is shown in figure 1. The trace displays a record of the movement of the peak of activity of the radiolabelled bolus along the tracheal mucosa. It consists of two phases: an initial phase during which the movement was linear representing a constant velocity of the bolus cephalad; the second phase of the plot is a plateau, corresponding to the bolus reaching the vocal cords.

In some studies the shape of the bolus changed during the 15-min acquisition period, dividing into separate, smaller boluses that subsequently re-coalesced. To ensure that this did not influence the measurement, separate measurements were obtained from three regions of the bolus (leading edge, trailing edge and peak of activity) in 38 studies of normal subjects and 21 studies of COPD patients. No significant differences were observed between the mean velocities for each region in the young normal subjects $(($ mean \pm SD) $10.0 \pm 4.2,10.7 \pm 4.6$ and $10.4 \pm 4.1 \mathrm{~mm} \cdot \mathrm{min}^{-1}$, respectively) or in the COPD patients $\left(1.5 \pm 2.4,1.3 \pm 2.5\right.$ and $1.4 \pm 2.4 \mathrm{~mm} \cdot \mathrm{min}^{-1}$, respectively) by ANOVA or by t-test ( $\mathrm{p}>0.05)$.

Analysis of repeated measurements of TMV, made on separate occasions in 13 young normal subjects and 16 COPD patients, by Bland-Altman plots (fig. 2) and coefficient of repeatability (10.3 and 5.6, respectively), indicated that measurements in normal subjects tended to be more variable than in COPD patients. The greatest variation occurred in 


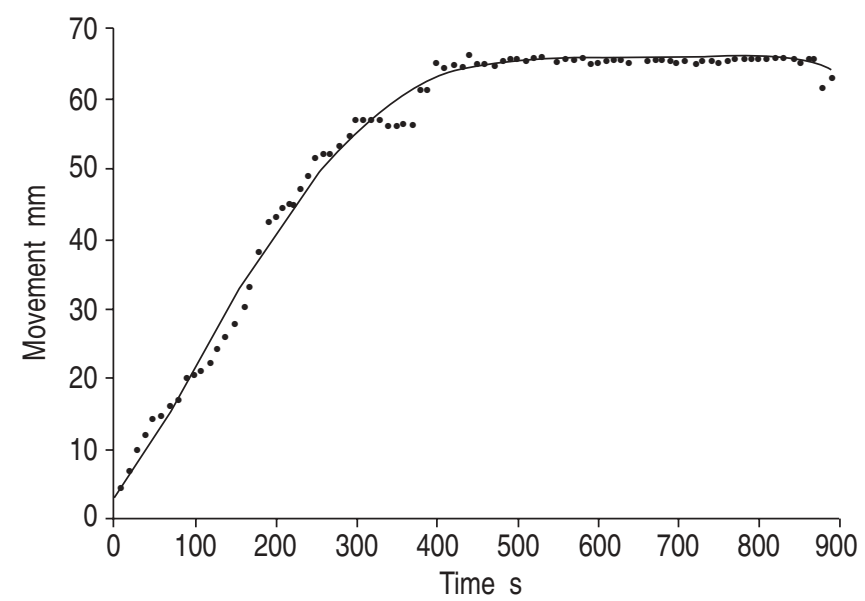

Fig. 1.-A representative tracheal mucus velocity (TMV) trace showing the movement of the peak of activity of the radiolabelled bolus along the tracheal mucosa of a normal subject. The positions of peak radioactivity were plotted as a function of time and a smoothed curve generated by application of a polynomial function to the data. The trace consists of two phases: an initial linear phase representing constant movement of the bolus cephalad; and a second plateau phase corresponding to the bolus reaching the vocal cords.

two normal subjects with a high mean TMV, suggesting that in some normal subjects there may be large fluctuations in mucociliary activity from day to day. The cause of this variability is not clear from these studies but may reflect subjects who had a stimulated mucociliary clearance on one of the occasions they were studied.

Figure 3 shows three representative TMV traces from patients with COPD as compared to a young normal subject. For COPD patients, the traces showed greatly reduced movement of the bolus.

Cough following the intra-tracheal injection occurred in only a few subjects. When the bolus was at or just below the vocal cords, cough sometimes resulted in movement of the bolus through the cords. When the bolus was further distal to

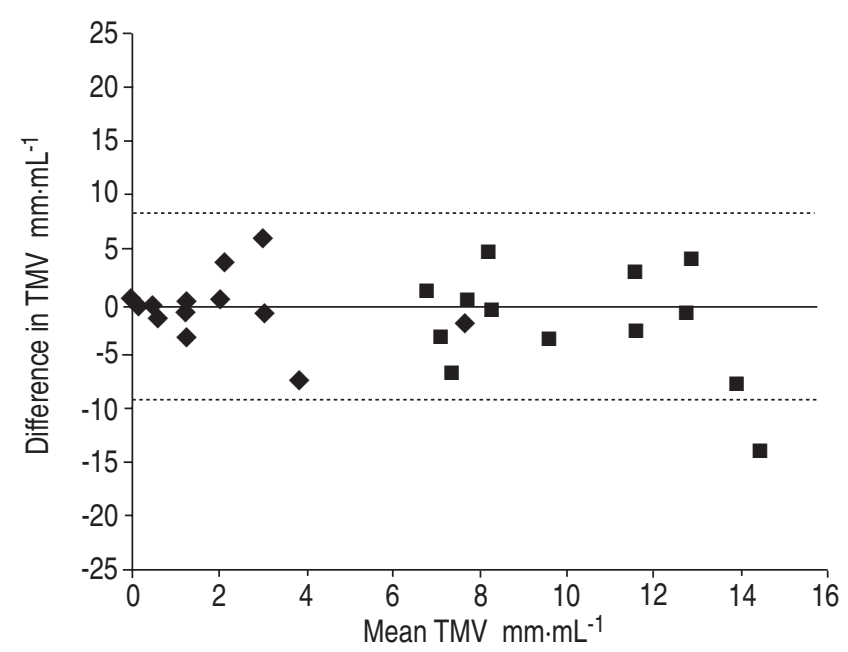

Fig. 2.-Bland-Altman plot showing the repeatability of tracheal mucus velocity (TMV) measurements in normal subjects $(n=13$; and in chronic obstructive pulmonary disease (COPD) patients $(n=16$; )). — : mean of the differences from the mean TMV, which in each case was the predicted value of zero; -----: coefficient of repeatability $( \pm 2 \mathrm{SD})$ of all studies (COPD and normal). As many of the COPD patients had very low TMV values, several data points overlapped.

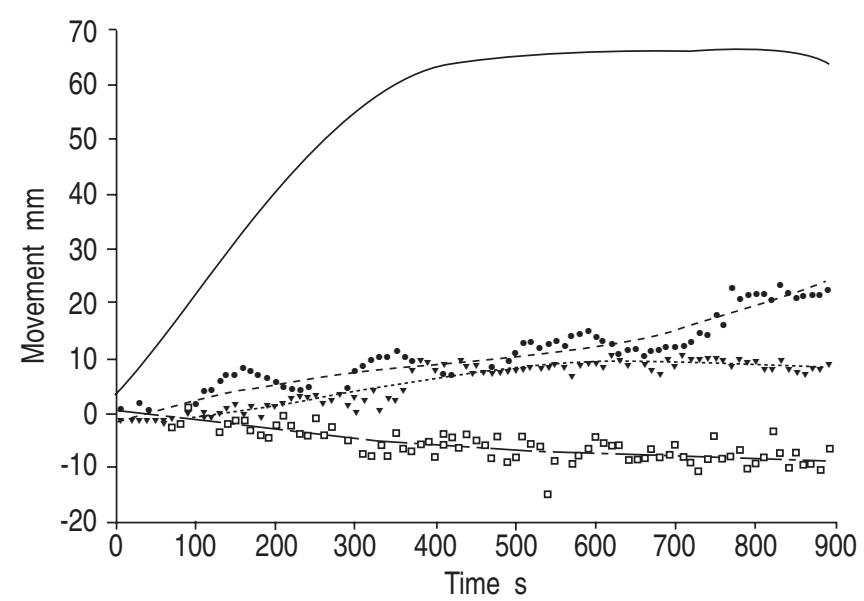

Fig. 3. - Representative tracheal mucus velocity (TMV) traces from three patients with chronic obstructive pulmonary disease (COPD 1: -; COPD 2: $\mathbf{\nabla}$; and COPD 3: $\square$ ), as compared to that of a normal subject (-). For COPD patients, the traces showed greatly reduced movement of the bolus. Most patients showed a very gradual cephalad movement of the bolus (COPD 2). In some cases (COPD 1) there was periodic cephalad and caudad movement with a net movement cephalad; in others, net movement of the bolus was caudad under the influence of gravity (COPD 3) suggesting no effective clearance.

the cords, no appreciable change in bolus position, peak activity or shape was observed.

The mean TMV in the young normal subjects was $10.7 \pm$ $3.5 \mathrm{~mm} \cdot \mathrm{min}^{-1}$. This was significantly greater than the mean TMV for older normal volunteers, $6.5 \pm 2.6 \mathrm{~mm} \cdot \mathrm{min}^{-1}(\mathrm{p}<0.001)$. In turn, TMV in COPD patients $\left(2.1 \pm 2.7 \mathrm{~mm} \cdot \mathrm{min}^{-1}\right)$ was significantly less $(\mathrm{p}<0.001)$ than in older normal subjects. As is shown in figure 4 .

\section{Discussion}

MCC is an important respiratory tract defence mechanism. The authors of this study have developed a rapid, safe and reproducible technique to measure TMV, which can be used to investigate abnormal clearance in disease and the

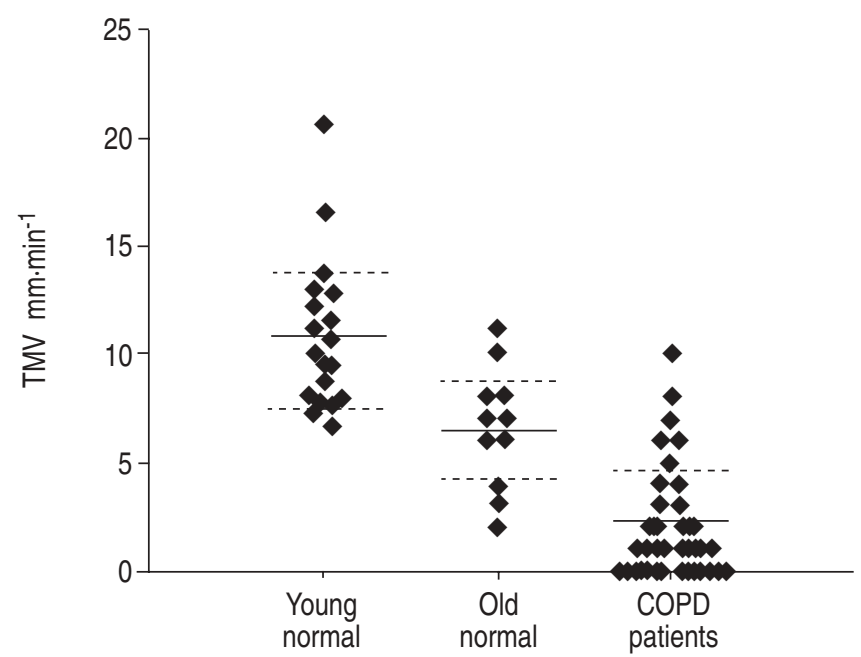

Fig. 4.-Scatter plot showing the range of tracheal mucus velocity (TMV) in normal subjects (young and old) and in chronic obstructive pulmonary disease (COPD) patients. - : mean TMV; ----: $\pm 1 \mathrm{SD}$. The means were all significantly different $(p<0.001)$ from each other. 
effectiveness of therapy. This technique represents a practical evolution of a methodology explored by several investigators in the past. It has several advantages over the more commonly used aerosol techniques of measuring lung clearance. In particular, it is rapid, requires ten-fold lower doses of ionising radiation, and label deposition and data analysis have been simplified. This measurement is limited to providing information from the trachea only and thus may not accurately reflect clearance throughout the tracheobronchial tree.

Various methods of droplet deposition were explored during the development of the technique. Initially, this was attempted through a fine catheter placed through the biopsy channel of a bronchoscope in a manner similar to that used by SANTA CRUZ et al. [10]. The bronchoscope was placed either just above or just below the vocal cords. However, this procedure was slow and poorly tolerated without sedation or local anaesthetic, both of which have the potential to impair MCC. Deposition of the droplet without provoking paroxysms of cough was critical and proved to be difficult with this approach. A further problem was the difficulty in controlling a small $(100 \mu \mathrm{L})$ droplet at the end of a long, narrow gauge catheter.

Cricothyroid injection is a simple procedure that is used commonly with lignocaine to produce topical anaesthesia prior to bronchoscopy. It was found that injection with a 25 gauge needle was well tolerated and caused minimal bruising. Most subjects did not cough and haemoptysis did not occur. In addition, it allowed a small volume of radiolabel to be handled easily. With practice, injection and immediate detection of the droplet was straightforward.

${ }^{99 \mathrm{~m}}$ Tc MAA was selected as the tracer since it is stable, not absorbed and possesses high specific activity. Furthermore, it is relatively inexpensive, readily available in kit form and widely used for other scintigraphic studies. The macroaggregates have a diameter of $10-40 \mu \mathrm{m}$, a particle size known to be cleared by MCC [11]. When deposited on the tracheal wall, the agent remains in droplet form on the mucus layer. Preparation, performance and analysis of the studies were not time consuming. Label preparation took $<5 \mathrm{~min}$, injection was completed in $<15 \mathrm{~s}$ and the images were acquired in $15 \mathrm{~min}$. Analysis is largely automated and a result can be obtained within 10 min after completion of the data acquisition. In the initial studies performed in normal subjects, the droplet was seen to move over a distance of 50-80 mm, which was adequate to allow analysis.

Both normal and COPD patients were comfortable lying still for the period of the test. Subject comfort is important in order to minimise body movement. By comparison, measuring MCC using inhaled radiolabelled aerosols and scintigraphy entails a prolonged acquisition time, during which the subject is required to lie supine for 45-60 min. Such standard aerosol studies also require active patient cooperation and training to ensure appropriate inhalation patterns and deposition of the aerosol.

On rare occasions, the deposition of the bolus elicited a cough response. However, in these subjects no movement of the bolus due to cough was observed. This lack of effective expulsion or increase in TMV following cough seems counterintuitive but has been reported previously [6]. The increase in MCC seen with cough in radiolabelled aerosol studies may reflect an effect on lower airways [12]. An absence of major effect of cough on TMV suggests that this technique may have increased utility in patient groups for whom the suppression of cough, even over 15 min, may be difficult (e.g. cystic fibrosis). In addition, this technique does not require any specific respiratory manoeuvres or breathing patterns.

This method of measuring TMV results in values for normal subjects in the same range (table 2) as those reported previously for the movement of insufflated teflon discs [8, 13], labelled albumin deposited directly into the trachea via bronchoscope [14] or by inhalation [5,6]. A comparison of techniques used to deposit the label (table 2) shows that the more invasive methods generally yielded higher TMV values, suggesting that the invasive methods may artifactually stimulate TMV. This technique, which is relatively noninvasive, represents a further evolution of earlier methodologies and provides results that are comparable to those published previously. There are some limitations to this technique. One is the potential anxiety evoked by cricothyroid injection. This proved to be a greater problem in recruiting healthy volunteers than patients, who accepted the procedure readily. However, even healthy volunteers were usually prepared to return for a second study. The anatomical landmarks that define a safe injection region are clear. A further potential limitation is that this method measures MCC in proximal trachea only. Proximal ciliated airways clear more quickly than distal airways [15]. Although MCC estimated by this technique may be faster than overall MCC, comparison within and between subjects would always be made from the same region.

This technique is not directly comparable to IRLA studies. IRLA studies can provide information on regional lung clearance, small airway ventilation and airway obstruction that are not possible by measurements of TMV. However, for measurement of large airway clearance, this technique has advantages over IRLA and previous methods of measuring TMV. It is more rapid, more comfortable and exposes subjects to ten-fold lower levels of radiation than required for a typical aerosol study. Resolution and image quality is not compromised by this dose minimisation. The authors have shown previously that standard scintillation detection systems can detect this very low level of activity and accurately track the movement of such a small bolus over small distances [16].

The information obtained by measuring TMV is complementary to that provided by IRLA studies. Further investigation is needed to determine the relationship between the different methods in normal subjects and patients with pulmonary disease.

It has been shown here that, in normal subjects, TMV was slower in a group aged $>50 \mathrm{yrs}\left(\right.$ mean $\left.\pm \mathrm{SD} 6.5 \pm 2.6 \mathrm{~mm} \cdot \mathrm{min}^{-1}\right)$ than a group aged $<50 \mathrm{yrs}\left(10.7 \pm 3.5 \mathrm{~mm} \cdot \mathrm{min}^{-1}\right)$. Other groups have reported a reduction in $\mathrm{MCC}$ of similar

Table 2.-Measurements of tracheal mucus velocity (TMV) (using various deposition techniques and radiolabelled markers

\begin{tabular}{lccr}
\hline First author [ref no.] & Marker & Deposition Route & TMV mm·min ${ }^{-1}$ \\
\hline GOODMAN [8] & Teflon discs & FOB & $10.1 \pm 3.5$ \\
CHOPRA [14] & $99 \mathrm{~m}$ Tc MAA & FOB & $15.5 \pm 0.7$ \\
TOOMES [13] & Teflon discs & FOB & $18.5 \pm 6.0$ \\
ZWAS [5] & $99 \mathrm{~m}$ Tc MAA & IRLA & $4.7 \pm 1.3$ \\
Current study & $99 \mathrm{~m}$ Tc MAA & CTI & $10.7 \pm 3.5$ \\
\hline
\end{tabular}

Data are presented as mean $\pm \mathrm{SD}$. ${ }^{99 \mathrm{~m}} \mathrm{Tc}$ MAA: Technetium-labelled macroaggregated human albumin; FOB: fibreoptic bronchoscopy; IRLA: inhaled radiolabelled aerosol; CTI: cricothyroid injection. 
magnitude with increasing age. GoODMAN et al. [8] showed that mean TMV in a group of young nonsmoking subjects (mean age $23 \mathrm{yrs}$ ) was $10.1 \mathrm{~mm} \cdot \mathrm{min}^{-1}$ and in a group of seven elderly nonsmokers (mean age $63 \mathrm{yrs}$ ) $5.8 \mathrm{~mm} \cdot \mathrm{min}^{-1}$. PUCHELLE et al. [17], using an inhaled radiolabelled aerosol technique, also demonstrated that clearance was reduced in older, as compared to younger, normal subjects [17].

Consistent with previous reports, impaired MCC $\left(2.1 \pm 2.7 \mathrm{~mm} \cdot \mathrm{min}^{-1}\right)$ in patients with COPD has been demonstrated here. GoODMAN et al. [8] showed that smokers and ex-smokers with simple and obstructive chronic bronchitis had markedly decreased TMV $\left(0.7 \pm 1.9\right.$ and $0.8 \mathrm{~mm} \cdot \mathrm{min}^{-1}$, respectively) compared to an age-matched nonsmoking group $\left(5.8 \pm 2.6 \mathrm{~mm} \cdot \mathrm{min}^{-1} ; \mathrm{p}<0.05\right)$. Several studies of MCC measured by IRLA have also reported impaired clearance in patients with COPD $[18,19]$. Putative disease-specific factors include the effects of cigarette smoke and recurrent infections. Possible mechanisms include progressive deciliation of airways [20, 21] and altered rheology of respiratory secretions [22]. Further studies are underway to determine whether TMV correlates with other clinical features of COPD (e.g. clinical progression, FEV1, smoking history).

A technique for measuring tracheal mucus velocity is reported here, which uses cricothyroid injection for deposition of a radiolabel and automated analysis of dynamic scintigraphic data. The technique is rapid, simple, safe and reproducible. It uses materials and methods that are widely available and relatively inexpensive. The relative simplicity of the technique should make measurements of tracheal mucus velocity more accessible to nonspecialist centres. This technique was used to measure tracheal mucus velocity in two groups of normal subjects and patients with chronic obstructive pulmonary disease. The results confirm that mucociliary clearance decreases with age and is markedly impaired in chronic obstructive pulmonary disease. This technique should facilitate evaluation of the role of abnormal clearance in disease, and the effects of time, acute infection and pharmacological intervention on mucociliary clearance.

\section{References}

1. Lourenco RV, Klimek MF, Borowski CJ. Deposition and clearance of 2 micron particles in the tracheobronchial tree of normal subjects - smokers and nonsmokers. J Clin Invest 1971; 50: 1411-1420.

2. Dorow P, Weiss T, Felix R, Schmutzler H. The influence of propranolol, metoprolol, and mepindolol on mucociliary clearance in coronary heart disease patients without pulmonary disease. Int J Clin Pharmacol Ther Toxicol 1984; 22: 108-111.

3. Matthys H, Kohler D. Effect of theophylline on mucociliary clearance in man. Eur J Respir Dis Suppl 1980; 109: 98-102.

4. Sackner MA, Rosen MJ, Wanner A. Estimation of tracheal mucous velocity by bronchofiberscopy. J Appl Physiol 1973; 34: 495-499.

5. Zwas ST, Katz I, Belfer B, Baum GL, Aharonson E.
Scintigraphic monitoring of mucociliary tracheobronchial clearance of technetium-99m macroaggregated albumin aerosol. J Nucl Med 1987; 28: 161-167.

6. Yeates DB, Aspin N, Levison $\mathrm{H}$, et al. Mucociliary tracheal transport rates in man. J Appl Physiol 1975; 39: 487-495.

7. Friedman M, Stott FD, Poole D, et al. A new roentgenographic method for estimating mucous velocity in airways. Am Rev Respir Dis 1977; 115: 67-72.

8. Goodman RM, Yergin BM, Landa JF, et al. Relationship of smoking history and pulmonary function tests to tracheal mucous velocity in nonsmokers, young smokers, ex-smokers, and patients with chronic bronchitis. Am Rev Respir Dis 1978; 117: 205-214.

9. Bland JM, Altman DG. Statistical methods for assessing agreement between two methods of clinical measurement. Lancet 1986; 1: 307-310.

10. Santa Cruz R, Landa J, Hirsch J, Sackner J. Tracheal mucous velocity in normal man and patients with obstructive lung disease. Effects of tertbutaline. Am Rev Resp Dis 1974; 109: 458-463.

11. Lippmann M, Leikauf G, Spektor D, et al. The effects of irritant aerosols on mucus clearance from large and small conductive airways. Chest 1981; 80: Suppl. 6, 873-877.

12. Camner P, Mossberg B, Afzelius BA. Measurements of tracheobronchial clearance in patients with immotile-cilia syndrome and its value in differential diagnosis. Eur J Respir Dis Suppl 1983; 127: 57-63.

13. Toomes H, Vogt-Moykopf I, Heller WD, Ostertag H. Measurement of mucociliary clearance in smokers and nonsmokers using a bronchoscopic video-technical method. Lung 1981; 159: 27-34.

14. Chopra SK. Measurement of tracheal mucociliary transport velocity in human smokers versus nonsmokers. Am Rev Respir Dis 1979; 119: Suppl. 205.

15. Morrow PE, Gibb FR, Gazioglu KM. A study of particulate clearance from the human lungs. Am Rev Respir Dis 1967; 96: 1209-1221.

16. Morgan LC, Pearson MA, Mackey DW, et al. Accuracy in gamma camera measurements of point source velocities. Nucl Med Commun 2000; 21: 553-556.

17. Puchelle E, Zahm JM, Bertrand A. Influence of age on bronchial mucociliary transport. Scand J Respir Dis 1979; 60: 307-313.

18. Lourenco RV. Distribution and clearance of aerosols. Am Rev Respir Dis 1970; 101: 460.

19. Vastag E, Matthys H, Kohler D, Gronbeck L, Daikeler G Mucociliary clearance and airways obstruction in smokers, ex-smokers and normal subjects who never smoked. Eur J Respir Dis Suppl 1985; 139: 93-100.

20. Auerbach O, Hammond EC, Garfinkel L. Changes in bronchial epithelium in relation to cigarette smoking, 1955-1960 vs 1970-1977. N Engl J Med 1979; 300: 381385.

21. Wanner A. A review of the effects of cigarette smoke on airway mucosal function. Eur J Respir Dis Suppl 1985; 139: 49-53.

22. King M, Wight A, De Sanctis G, et al. Mucus hypersecretion and viscoelasticity changes in cigarette-smoking dogs. Exp Lung Res 1989; 15: 375-389. 